\title{
Towards a better diagnosis of idiopathic pulmonary fibrosis
}

\author{
D. Valeyre
}

ABSTRACT: Idiopathic pulmonary fibrosis (IPF) is the most common of the idiopathic interstitial pneumonias, and poses significant clinical challenges. IPF diagnosis is based on clear-cut computed tomography (CT) and histopathological criteria, in an appropriate clinical context. The diagnostic criteria include: 1) exclusion of known causes of interstitial lung disease (including connective tissue disease); 2) usual interstitial pneumonia pattern on high-resolution CT in patients not subjected to surgical lung biopsy; and 3) specific combinations of high-resolution CT with pathological patterns in case of surgical lung biopsy. Improved diagnosis of IPF may help physicians to reduce the delay before an accurate diagnosis is made and increase patient awareness and access to adequate information, follow-up and treatment.

\section{KEYWORDS: Diagnosis, idiopathic pulmonary fibrosis}

diopathic interstitial pneumonias (IIPs) are a group of diseases also known as interstitial lung diseases (ILDs) in the absence of any known cause and of clinical manifestations other than limited to the lung [1]. Idiopathic pulmonary fibrosis (IPF) is the most common of the IIIs, accounting for $50-60 \%$ of diagnosed cases [2] and represents both the most frequent and most severe of all ILDs. Epidemiological studies suggest that IPF is more common in males with onset usually in middle or older age (prevalence peaks at age 65-79 yrs) [2], but it has no distinct geographic distribution and does not distinguish between particular races or ethnic groups [3]. It is usually sporadic but the notion of prior familial cases can be found in 3-10\% of cases. The main known risk factors that are associated with disease development include smoking (either current or past), some environmental factors, and genetic predisposition [3].

IPF is characterised by a distorted alveolarcapillary barrier architecture (several elements are involved including epithelial and endothelial cell apoptosis [4], infiltration of inflammatory cells into interstitial and alveolar spaces, fibroblast proliferation and excessive deposits of interstitial collagen) leading to an impaired gas exchange $[5,6]$. The specific molecular and cellular mechanisms, cause of disease onset and disease progression are still unknown. Despite some limits, animal models of pulmonary fibrosis can be of invaluable help for evidencing some of the pathogenic processes at play in IPF [7]. IPF is now considered as a distinct entity with lesions that vary in age and location. The established view that IPF was a disease in which fibrosis was directly caused by chronic inflammation has been challenged by two main arguments: 1) clinical measurements of inflammation failed to correlate with stage or outcome, and 2) potent anti-inflammatory therapy does not improve outcome [8].

The disease course in IPF is variable. Some patients may remain stable for long periods of time but a significant proportion demonstrate slow progression and others experience acute exacerbations leading to respiratory failure and death. In some patients, pulmonary hypertension may develop as a consequence of, or disproportionate to, the underlying lung disease and may explain a clinical deterioration despite preserved pulmonary lung function [9]. As a whole, median survival is estimated at 24-36 months and a 5-yr survival is found in $\leqslant 20 \%$ of patients.

IPF diagnosis is based on clear-cut computed tomography (CT) and pathological criteria in an appropriate clinical context [10]. However, diagnosing IPF in daily practice remains very challenging. There is often a long delay between the first manifestations of the disease and its accurate diagnosis. Moreover, an accurate diagnosis with a sufficient confidence level is not achieved in some cases. However, a correct diagnosis of IPF, as early as possible, may offer patients more optimal management [1].
CORRESPONDENCE

D. Valeyre

Dept of Pneumology

Hospital Avicenne

125 rue de Stalingrad

93009 Bobigny

France

E-mail: Dominique.valeyre@

avc.aphp.fr

Received:

Feb 142011

Accepted after revision:

April 042011

PROVENANCE

Publication of this peer-reviewed article was supported by InterMune Inc., Brisbane, CA, USA (article sponsor, European Respiratory Review issue 120). 


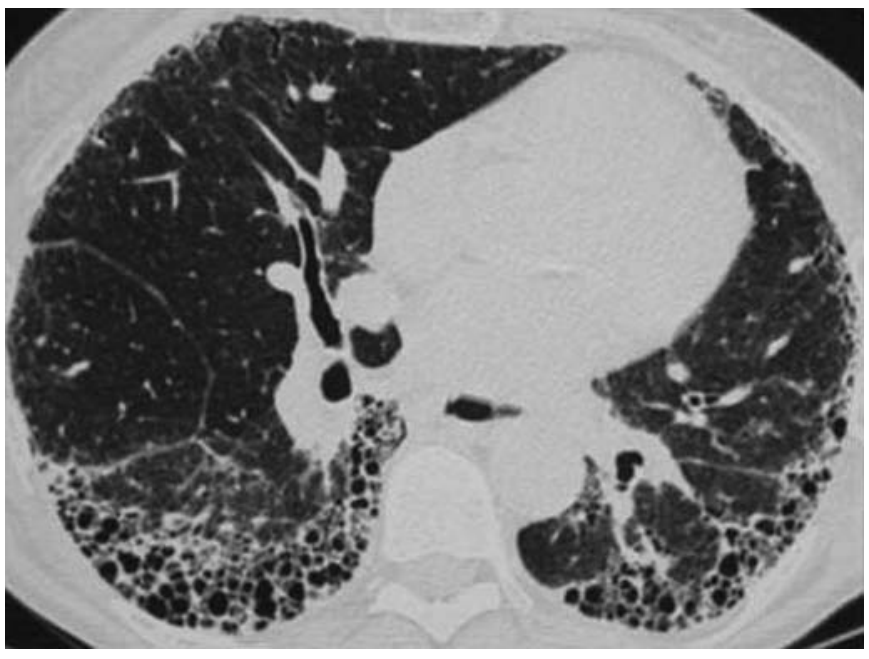

FIGURE 1. Usual interstitial pneumonia pattern on high-resolution computed tomography (M. Brauner, Dept of Radiology, Hospital Avicenne, Bobigny, France: personal communication).

\section{DIAGNOSTIC CRITERIA FOR IPF}

The first step, based on background investigation and physical examination, is to exclude known causes of ILD, e.g. certain drug toxicities, environmental exposures and connective tissue disease-associated ILD. Another important step based on thoracic CT and pathology consists of differentiating IPF from other IIPs, particularly nonspecific interstitial pneumonia (NSIP), but also desquamative interstitial pneumonia, respiratory bronchiolitis-associated ILD, acute interstitial pneumonia, cryptogenic organising pneumonia and lymphocytic interstitial pneumonia [1].

Findings on chest radiographs (CXR) of patients with IPF include peripheral reticular opacities that are most profuse at the lung bases [11]. However, several studies have evidenced the superior accuracy of high-resolution CT (HRCT) compared to CXR, since HRCT can identify abnormalities before they become visible on a CXR and confer more specificity [12], due to thin-section HRCT which increases spatial resolution and facilitates the visualisation of parenchymal detail to the level of the pulmonary lobules. The finding of a usual interstitial pneumonia (UIP) pattern on HRCT may be sufficient to diagnose IPF, with no need for surgical lung biopsy (SLB). A UIP pattern on HRCT relies on the following four criteria: 1) subpleural basal predominance; 2) reticular abnormality; 3) honeycombing with or without traction bronchiectasis; and 4) absence of features known to be inconsistent with the UIP pattern, e.g. condensations, nodules or pre-eminent ground glass (fig. 1) [13, 14]. If the observer is experienced, the accuracy of a diagnosis of IPF made according to HRCT criteria together with the clinical information is $\sim 90 \%$ [1]. In clinical practice, HRCT scanning can provide a confident, highly specific diagnosis for half to two-thirds of IPF patients [15] Moreover, the extent of disease observed on HRCT correlates with fibrosis and physiological impairment [16].

In the absence of a UIP pattern on HRCT, an SLB should be performed taking into consideration the patient's age, pulmonary function and absence of other specific contraindications. A typical UIP pattern at histopathology combines marked fibrosis with architectural distortion with or without honeycombing in a predominantly subpleural and paraseptal distribution, and patchy involvement of the lung parenchyma by fibroblast foci. Furthermore, the presence of small interstitial fibroblastic foci, frequently found at the periphery of remodelled areas, and the alternation of fibrosing areas and normal/subnormal parenchymal areas are two very important pathological criteria for the diagnosis. For a UIP pattern to be confirmed, features suggesting any alternative diagnosis must be lacking (fig. 2) [10]. If the patient has undergone SLB, the diagnosis will eventually rely on specific combinations of HRCT and pathological data evidencing more or less typical UIP patterns, respectively (table 1 ). This requires a multidisciplinary dynamic approach (pneumologist, radiologist and
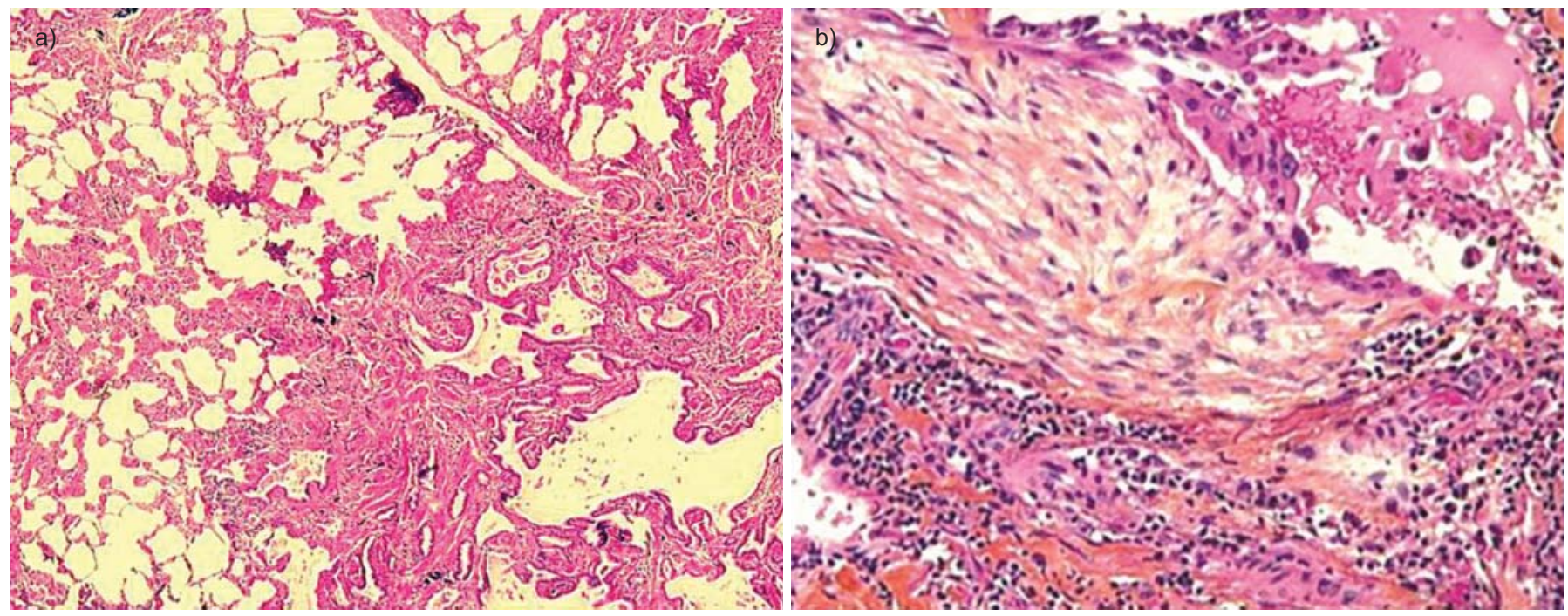

FIGURE 2. Usual interstitial pneumonia pattern on histopathology showing alternation of fibrosing areas and a) normal/subnormal parenchymal areas and b) fibroblastic foci (M. Kambouchner, Dept of Pathology, Hôpital Avicenne, Bobigny, France; personal communication). 


\begin{tabular}{|c|c|c|c|c|c|}
\hline TABLE 1 & $\begin{array}{l}\text { Diagnosis of idiopathic } p \\
\text { (HRCT) and histopatholo }\end{array}$ & $\begin{array}{l}\text { fibrosis bas } \\
\text { igations }\end{array}$ & oatterns dis & in high-resolution con & mography \\
\hline \multirow[t]{2}{*}{ HRCT pattern } & & \multicolumn{4}{|c|}{ Histopathology pattern } \\
\hline & UIP & Probable UIP & Possible UIP & Non-classifiable fibrosis & Not UIP \\
\hline UIP & Yes & Yes & Yes & Yes & No \\
\hline
\end{tabular}

pathologist expert on ILD) which has been shown to improve diagnosis $[17,18]$.

\section{CHALLENGES IN DIAGNOSING IPF IN DAILY PRACTICE Delay in diagnosis}

Based on informal surveys and the author's own clinical experience, some preliminary observations may be made regarding the timeliness of IPF diagnosis. Suspicion of IPF too often arises several months after the first manifestations of the disease. Typically, the first clinical features that may suggest IPF (subtle cough and/or dyspnoea) are often overlooked in the context of past or current smoking habits and/or ageing. During this phase, the physician often fails to suspect that the earlier manifestations could be due to IPF. Another important cause of delay in IPF diagnosis is secondary to the behaviour of some physicians who consider it good practice to make an approximate diagnosis of fibrosing pneumonitis, on the grounds that more differentiation among IIP will not significantly impact on the care of patients. At present, the proportion of patients with late-diagnosed IPF has not been well studied, either within or across national health systems.

\section{The difficulty of confirming a diagnosis}

Although diagnostic guidelines are available [1] they may not be consistently applied by physicians for a number of reasons. For example, the patient may not be investigated thoroughly enough to rule out alternative diagnoses. Exposure to causes of ILD simulating IPF on HRCT can be overlooked. The absence of a cautious physical examination can lead a physician to fail to recognise a connective tissue disease. For instance, without an adequate review of a patient's medical history and past documents a stage IV sarcoidosis could be overlooked, as in rare cases the CT scan can reveal honeycombing mimicking a basal and peripheral UIP pattern [19-21]. The CT and biopsy specimens may not be of a sufficient quality to confirm the diagnosis. The radiologist and/or the pathologist may be insufficiently experienced. The omission of multidisciplinary discussion $[17,18]$ may lead to failure to identify IPF. Finally, subjective findings of HRCT such as honeycombing may show significant inter-observer variability, making the diagnosis difficult to confirm. It is important to emphasise that radiologists with differing levels of experience and expertise may interpret radiographic images differently. A multinational European study has found that although the overall accuracy of a clinical diagnosis of IPF in expert centres is good (87.2\%), the level of agreement within the expert panels that assessed the diagnoses was only fair to moderate [22]. Thus, it is essential to have a multidisciplinary discussion with a pneumologist, a radiologist and a pathologist who are familiar with ILD.

\section{THE NEED FOR EARLY DIAGNOSIS}

Early diagnosis of IPF offers benefits to patients, including adequate information, lung transplantation enrolment, avoidance of inappropriate drugs (e.g. steroids and immunosuppressives), and access to trials and new treatments. Healthcare providers should, therefore, be mindful of IPF as a potential diagnosis particularly in newly presenting patients who are aged $>55$ yrs and in patients who are smokers, ensuring that initial symptoms such as cough and dyspnoea are not considered as nonsignificant, but instead trigger the appropriate investigations. Moreover, faced with the aetiological diagnosis of ILD, IPF (the most frequent ILD) has to be considered as one of the most probable hypotheses, particularly in patients aged $>55$ yrs, according to Bayes' theorem.

\section{IMPROVING DIAGNOSIS}

\section{The pillars of an improved IPF diagnosis}

The guidelines (exclusion of alternate diagnosis, combination CT and histopathological evaluation, and multidisciplinary discussion) have to be strictly applied. HRCT sensitivity may also be enhanced if the scan is performed with the patient in the prone position. The availability of validated clinical diagnosis predictors could help to determine the accurate estimation of IPF diagnosis probability at an individual level, allowing a more confident diagnosis and reducing the need for pulmonary biopsy, as suggested by the very convincing study by Fell et al. [23]. However, confirmation of the results has to be obtained from further series before application in clinical practice.

Some technical improvements could arise from the availability of new tools, although currently there is no evidence for such a contribution. Post-processing tools could help differentiate between honeycombing and bronchiectasis when both are discussed on HRCT [24]. Computer-aided diagnosis (threshold segmentation method or texture analysis) may also be a new useful contribution for the future [25].

Even though they are no longer part of the main IPF diagnosis recommendations, both bronchoalveolar lavage (BAL) and pulmonary function testing remain interesting tools for specific conditions. 


\section{TABLE 2 Candidate biomarkers for idiopathic pulmonary fibrosis (IPF)}

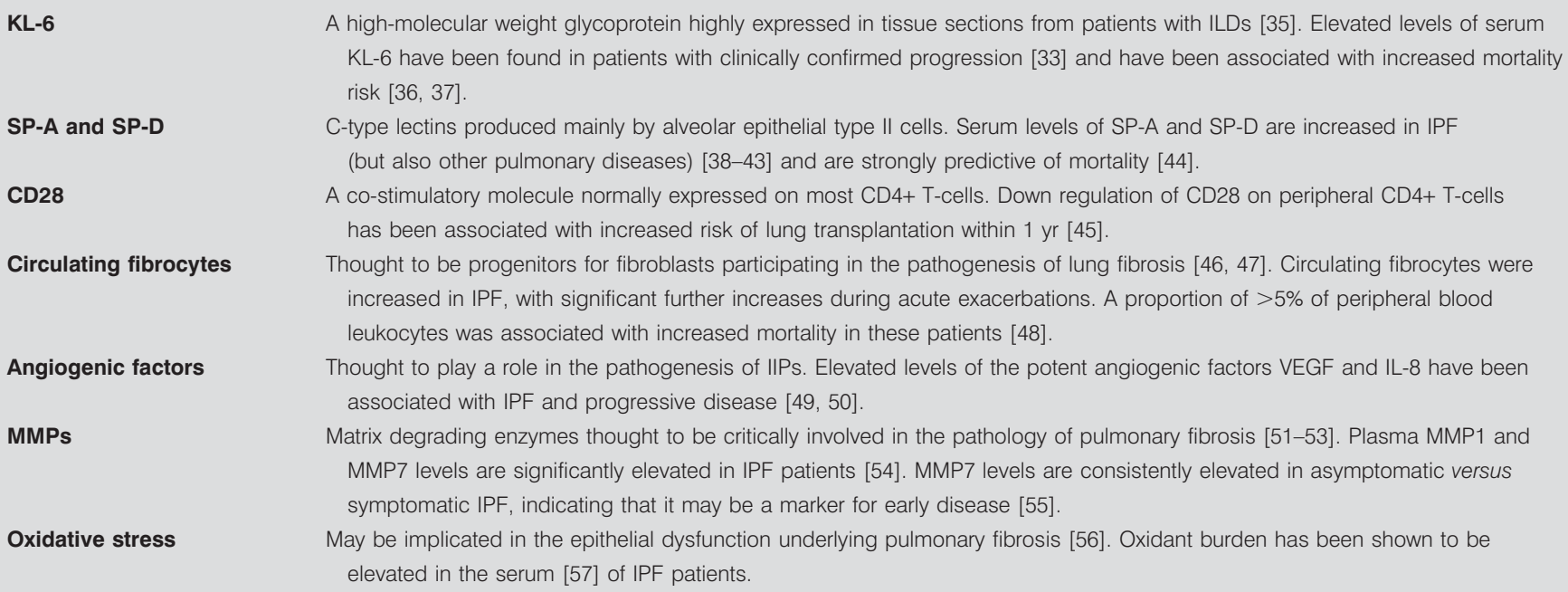

A high-molecular weight glycoprotein highly expressed in tissue sections from patients with ILDs [35]. Elevated levels of serum KL-6 have been found in patients with clinically confirmed progression [33] and have been associated with increased mortality risk [36, 37].

C-type lectins produced mainly by alveolar epithelial type II cells. Serum levels of SP-A and SP-D are increased in IPF (but also other pulmonary diseases) [38-43] and are strongly predictive of mortality [44].

A co-stimulatory molecule normally expressed on most CD4+ T-cells. Down regulation of CD28 on peripheral CD4+ T-cells has been associated with increased risk of lung transplantation within $1 \mathrm{yr}$ [45]

Thought to be progenitors for fibroblasts participating in the pathogenesis of lung fibrosis [46, 47]. Circulating fibrocytes were increased in IPF, with significant further increases during acute exacerbations. A proportion of $>5 \%$ of peripheral blood leukocytes was associated with increased mortality in these patients [48].

Thought to play a role in the pathogenesis of IIPs. Elevated levels of the potent angiogenic factors VEGF and IL-8 have been associated with IPF and progressive disease [49, 50].

Matrix degrading enzymes thought to be critically involved in the pathology of pulmonary fibrosis [51-53]. Plasma MMP1 and MMP7 levels are significantly elevated in IPF patients [54]. MMP7 levels are consistently elevated in asymptomatic versus symptomatic IPF, indicating that it may be a marker for early disease [55].

May be implicated in the epithelial dysfunction underlying pulmonary fibrosis [56]. Oxidant burden has been shown to be elevated in the serum [57] of IPF patients.

KL-6: Krebs von Lungen factor-6; SP: surfactant protein; MMP; matrix metalloproteinase; ILD: interstitial lung disease; IIP: idiopathic interstitial pneumonia; VEGF: vascular endothelial growth factor; IL: interleukin.

The value of BAL in excluding other disorders in the American Thoracic Society/European Respiratory Society algorithm for the diagnosis of IPF needs to be evaluated [26, 27]. However, BAL findings support the ruling out of other potential differential diagnoses, such as hypersensitivity pneumonitis, through the demonstration of a lymphocytosis $>30 \%$. In the follow-up of patients with IPF, BAL is indicated whenever new infiltrates develop suggesting infection or acute exacerbation of IPF. However, serial BAL to monitor the course of disease cannot be routinely recommended [28].

Serial changes in pulmonary function tests (PFTs) at 6 or 12 months have greater prognostic value than baseline data [1, 29-33]. "Significant decline" is defined as a reduction from baseline values of $10 \%$ for forced vital capacity and $15 \%$ for diffusing capacity of the lung for carbon monoxide (DL,CO). "Marginal declines" (i.e. declines that do not meet the threshold value) may nevertheless indicate real disease progression, especially when accompanied by increased symptoms or other evidence [34]. ZAPPALA et al. [34] have remarked that if real disease progression could be defined in terms of marginal PFT thresholds, then this would allow increased recognition of clinically relevant disease behaviour. For example, evolution of pulmonary function as evidenced by PFTs may assist in differential diagnosis, with significant or marginal declines of forced vital capacity $(>5 \%)$ and $D L, C O(>7.5 \%)$ being significantly more common in IPF, compared to NSIP [34].

Biomarkers are a potentially valuable tool in diagnosing and treating patients with IPF. However, currently identified candidate biomarkers (table 2) present a number of drawbacks. Their specificity for a single interstitial lung disease is poor. Most have been tested only in limited numbers of patients and have not been prospectively validated. In addition, it is not yet clear whether they will provide useful information in addition to that provided by existing tests. For example, Krebs von Lungen factor-6 (KL-6) seems to be a good surrogate marker for pulmonary fibrosis, but at present cannot replace conventional diagnostic procedures [58]. Although it appears unlikely that a biomarker alone will become a valuable diagnostic tool, a combination of several biomarkers may be a promising direction for research. For example, the composite measurement of five serum proteins has been shown to correctly differentiate between IPF patients and controls, with a sensitivity of $98.6 \%$ and specificity of $98.1 \%[55,58]$.

\section{CONCLUSION}

IPF is a severe condition with a worse prognosis than all other ILDs. The accuracy and timeliness of IPF diagnosis must be improved in order to improve treatment opportunities and outcomes. However, there is often a long delay before a diagnosis is made and today the diagnosis is too often insufficiently secure or accurate. In order to achieve this goal of a better diagnosis, a range of issues need to be addressed. The guidelines (exclusion of alternate diagnosis, optimal interpretation of $\mathrm{CT}$, combination $\mathrm{CT}$ and histopathological evaluation with a multidisciplinary discussion implying a pulmonologist, a radiologist and a pathologist expert on ILD) have to be strictly applied. Improvements could come from the availability of more accurate and validated diagnosis predictors, new tools optimising CT efficacy and new more specific biomarkers.

\section{STATEMENT OF INTEREST}

D. Valeyre has participated as an investigator and/or member of a steering committee in several trials on idiopathic pulmonary fibrosis (INSPIRE, CAPACITY, BUILD1, BUILD3, MUSIC and BIBF).

\section{ACKNOWLEDGEMENTS}

This article is based on the proceedings of a satellite symposium held at the 2010 ERS Annual Congress (Barcelona, Spain), which was sponsored by InterMune Inc. The author was assisted in the preparation of the text by professional medical writers at IntraMed International (Milan, Italy). The medical writing support was funded by InterMune Inc. 


\section{REFERENCES}

1 American Thoracic Society, European Respiratory Society. American Thoracic Society/European Respiratory Society International Multidisciplinary Consensus Classification of the Idiopathic Interstitial Pneumonias. Am J Respir Crit Care Med 2002; 165: 277-304.

2 Raghu G, Weycker D, Edelsberg J, et al. Incidence and prevalence of idiopathic pulmonary fibrosis. Am J Respir Crit Care Med 2006; 174: 810-816.

3 American Thoracic Society. Idiopathic pulmonary fibrosis: diagnosis and treatment. International consensus statement. American Thoracic Society (ATS), and the European Respiratory Society (ERS). Am J Respir Crit Care Med 2000; 161: 646-664.

4 Kuwano K, Hagimoto N, Nakanishi Y. The role of apoptosis in pulmonary fibrosis. Histol Histopathol 2004; 19: 867-881.

5 Thannickal VJ, Horowitz JC. Evolving concepts of apoptosis in idiopathic pulmonary fibrosis. Proc Am Thorac Soc 2006; 3: 350-356.

6 Strieter RM. Pathogenesis and natural history of usual interstitial pneumonia: the whole story or the last chapter of a long novel. Chest 2005; 128: Suppl. 1, 526S-532S.

7 Avcuoglu S, Wygrecka M, Marsh LM, et al. The TrkB/NT4 signaling axis is perturbed in clinical and experimental pulmonary fibrosis. Am J Respir Cell Mol Biol 2011; [Epub ahead of print DOI: 10.1165/rcmb.2010-0195OC].

8 Noble PW, Homer RJ. Back to the future: historical perspective on the pathogenesis of idiopathic pulmonary fibrosis. Am J Respir Cell Mol Biol 2005; 33: 113-120.

9 Corte TJ, Wort SJ, Wells AU. Pulmonary hypertension in idiopathic pulmonary fibrosis: a review. Sarcoidosis Vasc Diffuse Lung Dis 2009; 26: 7-19.

10 Raghu G. The development of evidence-based guidelines of the ATS, ERS, JRS and ALAT Joint Task Force for the management of IPF: new diagnostic criteria, natural course and approach to the diagnosis of IPF. 2010 American Thoracic Society Congress, New Orleans, USA. B82.

11 Martinez FJ, Safrin S, Weycker D, et al. The clinical course of patients with idiopathic pulmonary fibrosis. Ann Intern Med 2005; 142: 963-967.

12 Aziz ZA, Wells AU, Bateman ED, et al. Interstitial lung disease: effects of thin-section CT on clinical decision making. Radiology 2006; 238: 725-733.

13 Schmidt SL, Sundaram B, Flaherty KR. Diagnosing fibrotic lung disease: when is high-resolution computed tomography sufficient to make a diagnosis of idiopathic pulmonary fibrosis? Respirology 2009; 14: 934-939.

14 Sumikawa H, Johkoh T, Colby TV, et al. Computed tomography findings in pathological usual interstitial pneumonia: relationship to survival. Am J Respir Crit Care Med 2008; 177: 433-439.

15 Gotway MB, Freemer MM, King TE Jr. Challenges in pulmonary fibrosis. 1: Use of high resolution CT scanning of the lung for the evaluation of patients with idiopathic interstitial pneumonias. Thorax 2007; 62: 546-553.

16 Noth I, Martinez FJ. Recent advances in idiopathic pulmonary fibrosis. Chest 2007; 132: 637-650.

17 Flaherty KR, King TE Jr, Raghu G, et al. Idiopathic interstitial pneumonia: what is the effect of a multidisciplinary approach to diagnosis? Am J Respir Crit Care Med 2004; 170: 904-910.

18 Flaherty KR, Andrei AC, King TE Jr, et al. Idiopathic interstitial pneumonia: do community and academic physicians agree on diagnosis? Am J Respir Crit Care Med 2007; 175: 1054-1060.

19 Abehsera M, Valeyre D, Grenier P, et al. Sarcoidosis with pulmonary fibrosis: CT patterns and correlation with pulmonary function. AJR Am J Roentgenol 2000; 174: 1751-1757.

20 Padley SP, Padhani AR, Nicholson A, et al. Pulmonary sarcoidosis mimicking cryptogenic fibrosing alveolitis on CT. Clin Radiol 1996; 51: 807-810.
21 Shigemitsu H, Oblad JM, Sharma OP, et al. Chronic interstitial pneumonitis in end-stage sarcoidosis. Eur Respir J 2010; 35: 695697.

22 Thomeer M, Demedts M, Behr J, et al. Multidisciplinary interobserver agreement in the diagnosis of idiopathic pulmonary fibrosis. Eur Respir J 2008; 31: 585-591.

23 Fell CD, Martinez FJ, Liu LX, et al. Clinical predictors of a diagnosis of idiopathic pulmonary fibrosis. Am J Respir Crit Care Med 2010; 181: 832-837.

24 Beigelman-Aubry C, Hill C, Guibal A, et al. Multi-detector row CT and postprocessing techniques in the assessment of diffuse lung disease. Radiographics 2005; 25: 1639-1652.

25 Boehm HF, Fink C, Attenberger U, et al. Automated classification of normal and pathologic pulmonary tissue by topological texture features extracted from multi-detector CT in 3D. Eur Radiol 2008; 18: $2745-2755$.

26 Wells AU. The clinical utility of bronchoalveolar lavage in diffuse parenchymal lung disease. Eur Respir Rev 2010; 19: 237-241.

27 Harari S, Caminati A. Update on diffuse parenchymal lung disease. Eur Respir Rev 2010; 19: 97-108.

28 Kinder BW, Brown KK, Schwarz MI, et al. Baseline BAL neutrophilia predicts early mortality in idiopathic pulmonary fibrosis. Chest 2008; 133: 226-232.

29 Latsi PI, du Bois RM, Nicholson AG, et al. Fibrotic idiopathic interstitial pneumonia: the prognostic value of longitudinal functional trends. Am J Respir Crit Care Med 2003; 168: 531-537.

30 Flaherty KR, Andrei AC, Murray S, et al. Idiopathic pulmonary fibrosis: prognostic value of changes in physiology and sixminute-walk test. Am J Respir Crit Care Med 2006; 174: 803-809.

31 Collard HR, King TE Jr, Bartelson BB, et al. Changes in clinical and physiologic variables predict survival in idiopathic pulmonary fibrosis. Am J Respir Crit Care Med 2003; 168: 538-542.

32 Jegal Y, Kim DS, Shim TS, et al. Physiology is a stronger predictor of survival than pathology in fibrotic interstitial pneumonia. Am J Respir Crit Care Med 2005; 171: 639-644.

33 Hanson D, Winterbauer $\mathrm{RH}$, Kirtland $\mathrm{SH}$, et al. Changes in pulmonary function test results after 1 year of therapy as predictors of survival in patients with idiopathic pulmonary fibrosis. Chest 1995; 108: 305-310.

34 Zappala CJ, Latsi PI, Nicholson AG, et al. Marginal decline in forced vital capacity is associated with a poor outcome in idiopathic pulmonary fibrosis. Eur Respir J 2010; 35: 830-836.

35 Kohno N, Kyoizumi S, Awaya Y, et al. New serum indicator of interstitial pneumonitis activity. Sialylated carbohydrate antigen KL-6. Chest 1989; 96: 68-73.

36 Satoh H, Kurishima K, Ishikawa H, et al. Increased levels of KL-6 and subsequent mortality in patients with interstitial lung diseases. J Intern Med 2006; 260: 429-434.

37 Yokoyama A, Kondo K, Nakajima M, et al. Prognostic value of circulating KL-6 in idiopathic pulmonary fibrosis. Respirology 2006; 11: 164-168.

38 Doyle IR, Nicholas TE, Bersten AD. Serum surfactant protein-A levels in patients with acute cardiogenic pulmonary oedema and adult respiratory distress syndrome. Am J Respir Crit Care Med 1995; 152: 307-317.

39 Greene KE, King TE Jr, Kuroki Y, et al. Serum surfactant proteins-A and -D as biomarkers in idiopathic pulmonary fibrosis. Eur Respir J 2002; 19: 439-446.

40 Kuroki Y, Takahashi H, Chiba H, et al. Surfactant proteins A and D: disease markers. Biochim Biophys Acta 1998; 1408: 334-345.

41 Kuroki Y, Tsutahara S, Shijubo N, et al. Elevated levels of lung surfactant protein $\mathrm{A}$ in sera from patients with idiopathic pulmonary fibrosis and pulmonary alveolar proteinosis. Am Rev Respir Dis 1993; 147: 723-729.

42 Ishii $\mathrm{H}$, Mukae $\mathrm{H}$, Kadota J, et al. High serum concentrations of surfactant protein $\mathrm{A}$ in usual interstitial pneumonia compared with non-specific interstitial pneumonia. Thorax 2003; 58: 52-57. 
43 Cheng IW, Ware LB, Greene KE, et al. Prognostic value of surfactant proteins $\mathrm{A}$ and $\mathrm{D}$ in patients with acute lung injury. Crit Care Med 2003; 31: 20-27.

44 Kinder BW, Brown KK, McCormack FX, et al. Serum surfactant protein-A is a strong predictor of early mortality in idiopathic pulmonary fibrosis. Chest 2009; 135: 1557-1563.

45 Gilani SR, Vuga LJ, Lindell KO, et al. CD28 down-regulation on circulating CD4 T-cells is associated with poor prognoses of patients with idiopathic pulmonary fibrosis. PLoS One 2010; 5: e8959.

46 Mehrad B, Burdick MD, Zisman DA, et al. Circulating peripheral blood fibrocytes in human fibrotic interstitial lung disease. Biochem Biophys Res Commun 2007; 353: 104-108.

47 Pilling D, Roife D, Wang M, et al. Reduction of bleomycin-induced pulmonary fibrosis by serum amyloid P. J Immunol 2007; 179: 4035-4044.

48 Moeller A, Gilpin SE, Ask K, et al. Circulating fibrocytes are an indicator of poor prognosis in idiopathic pulmonary fibrosis. Am J Respir Crit Care Med 2009; 179: 588-594.

49 Ebina M, Shimizukawa M, Shibata N, et al. Heterogeneous increase in CD34-positive alveolar capillaries in idiopathic pulmonary fibrosis. Am J Respir Crit Care Med 2004; 169: 1203-1208.

50 Simler NR, Brenchley PE, Horrocks AW, et al. Angiogenic cytokines in patients with idiopathic interstitial pneumonia. Thorax 2004; 59: 581-585.
51 Henry MT, McMahon K, Mackarel AJ, et al. Matrix metalloproteinases and tissue inhibitor of metalloproteinase- 1 in sarcoidosis and IPF. Eur Respir J 2002; 20: 1220-1227.

52 Suga M, Iyonaga K, Okamoto T, et al. Characteristic elevation of matrix metalloproteinase activity in idiopathic interstitial pneumonias. Am J Respir Crit Care Med 2000; 162: 1949-1956.

53 Huh JW, Kim DS, Oh YM, et al. Is metalloproteinase-7 specific for idiopathic pulmonary fibrosis? Chest 2008; 133: 1101-1106.

54 Selman M, Pardo A, Barrera L, et al. Gene expression profiles distinguish idiopathic pulmonary fibrosis from hypersensitivity pneumonitis. Am J Respir Crit Care Med 2006; 173: 188-198.

55 Rosas IO, Richards TJ, Konishi K, et al. MMP1 and MMP7 as potential peripheral blood biomarkers in idiopathic pulmonary fibrosis. PLoS Med 2008; 5: e93.

56 Kliment CR, Oury TD. Oxidative stress, extracellular matrix targets, and idiopathic pulmonary fibrosis. Free Radic Biol Med 2010; 49: 707-717.

57 Daniil ZD, Papageorgiou E, Koutsokera A, et al. Serum levels of oxidative stress as a marker of disease severity in idiopathic pulmonary fibrosis. Pulm Pharmacol Ther 2008; 21: 26-31.

58 van den Blink B, Wijsenbeek MS, Hoogsteden HC. Serum biomarkers in idiopathic pulmonary fibrosis. Pulm Pharmacol Ther 2010; 23: 515-520. 